\title{
Prognostic impact of activated B-cell focused classification in diffuse large B-cell lymphoma patients treated with R-CHOP
}

\author{
Heidi Nyman ${ }^{1,2}$, Mats Jerkeman ${ }^{3}$, Marja-Liisa Karjalainen-Lindsberg ${ }^{4}$, Alison H Banham ${ }^{5}$ \\ and Sirpa Leppä ${ }^{1,2}$ \\ ${ }^{1}$ Department of Oncology, Helsinki University Central Hospital, Helsinki, Finland; ${ }^{2}$ Molecular Cancer Biology \\ Program, Biomedicum Helsinki, University of Helsinki, Helsinki, Finland; ${ }^{3}$ Department of Oncology, Lund \\ University Hospital, Lund, Sweden; ${ }^{4}$ Department of Pathology, Haartman Institute, University of Helsinki, \\ Helsinki, Finland and ${ }^{5}$ Nuffield Department of Clinical Laboratory Sciences, University of Oxford, John \\ Radcliffe Hospital, Oxford, UK
}

\begin{abstract}
Gene expression profiling studies initially enabled diffuse large B-cell lymphoma to be divided into germinal center and activated B-cell-like subtypes, which define high- and low-risk patient groups when treated with chemotherapy. Attempts to reproduce the prognostic classification immunohistochemically have, however, provided inconsistent results. The aim of this study was to determine whether modified immunohistochemical classification of cell of origin focusing on activated B-cell-like markers could be used to predict the outcome of immunochemotherapy-treated diffuse large B-cell lymphoma patients. The expression of CD10, Bcl-6, MUM1/ IRF4, Bcl-2, and FOXP1 was determined immunohistochemically from 88 samples of diffuse large B-cell lymphoma patients treated uniformly with R-CHOP. When the modified classification using MUM1/IRF4 and FOXP1 positivities as activated B-cell-like markers was applied to distinguish the patients between the activated B-cell-like and other diffuse large B-cell lymphoma subtypes, a significantly worse outcome was seen for the patients with the activated B-cell-like phenotype (3-year failure-free survival 63 vs $82 \%, P=0.048$, overall survival 69 vs $85 \%, P=0.110$ ). Similarly, according to the Muris algorithm, the group 2 patients representing Bcl-2-positive post-germinal center patients showed an inferior outcome in comparison to the group 1 patients (failure-free survival 59 vs $81 \%, P=0.041$, overall survival 67 vs $82 \%, P=0.159$ ). In contrast, when the classification of the same cohort was performed according to the Hans algorithm, no significant difference in survival was observed between the germinal center and non-germinal center patients. In conclusion, the data suggest that both the modified activated B-cell-like and Muris classifications define the non-germinal center phenotype as an adverse risk factor in R-CHOP-treated diffuse large B-cell lymphoma patients.
\end{abstract}

Modern Pathology (2009) 22, 1094-1101; doi:10.1038/modpathol.2009.73; published online 15 May 2009

Keywords: diffuse large B-cell lymphoma; MUM1/IRF4; activated B-cell-like; R-CHOP; prognosis

Diffuse large B-cell lymphoma is the most common lymphoma subtype, accounting for $30 \%$ of all nonHodgkin's lymphomas. It shows an aggressive clinical course, and comprises a heterogeneous group of lymphomas in terms of morphology, immunophenotype and molecular abnormalities. ${ }^{1}$ Currently, a combination of the anti-CD20 antibody, rituximab, with an antracyclin-based chemotherapy

Correspondence: Dr H Nyman, MD, Department of Oncology, Helsinki University Central Hospital, P.O. Box 180, FIN-00029 HUS, Finland.

E-mail: heidi.nyman@helsinki.fi

Received 6 February 2009; revised 8 April 2009; accepted 16 April 2009; published online 15 May 2009 regimen (immunochemotherapy) is accepted as a standard first-line treatment, ${ }^{2-5}$ and depending on the clinical and biological features $50-70 \%$ of the patients can be cured.

Based on gene expression profiles diffuse large B-cell lymphoma can be divided into molecular subtypes. Two major subgroups, displaying germinal center B-cell and activated B-cell-like signatures, have been identified. ${ }^{6,7}$ The classification has prognostic significance in response to chemotherapy, as the patients with the germinal center signature have a better survival compared to the patients with the activated B-cell-like signature. According to recent data, the prognostic impact of this gene expression-based classification is not 
affected by the addition of rituximab to chemotherapy. ${ }^{8-10}$ Confirmation of these findings remains, however, limited and gene expression technology is not currently available for routine clinical use.

As immunohistochemical classification of cell of origin would be more practical than the gene expression-based methods, several attempts have been performed to identify the germinal center and non-germinal center subgroups of diffuse large B-cell lymphoma immunohistochemically. The most widely applied Hans algorithm based on immunostaining for CD10, Bcl-6, and MUM1/IRF4 reproduces the gene expression-based classification of germinal center and non-germinal center subtypes with a misclassification rate of $20 \% .{ }^{11}$ According to some studies, immunohistochemically defined germinal center patients have a significantly better survival than the non-germinal center patients, ${ }^{11-13}$ whereas in other studies no differences in the survival rates have been seen. ${ }^{14-16}$ In the immunochemotherapy era the results have also been inconsistent. For example, we and others found no significant correlation between the cell of origin and outcome in the patients treated with immunochemotherapy. ${ }^{17-22}$ In contrast, $\mathrm{Fu}$ et $a l^{23}$ reported a trend towards a better event-free survival and a significantly better overall survival for the patients with the germinal center phenotype as compared with the non-germinal center group of patients.

Another approach to immunohistochemically define subgroups of diffuse large B-cell lymphoma has been recently reported by Muris et al ${ }^{24}$ Based on the expression of Bcl-2, CD10, and MUM1/IRF4 the algorithm defines the Bcl-2-positive, CD10negative, and MUM1/IRF4-positive immunophenotype (group 2) as an adverse risk factor for chemotherapy-treated patients. However, the utility of the algorithm needs to be confirmed in the rituximab-era.

Both biological and technical factors are likely to account for the inconsistent results obtained in the immunohistochemical studies. Biological factors include patient- and treatment-related features, such as the heterogeneity of patient populations and variable treatment regimens. Technical factors in turn are related to technical variations in staining protocols, the differences in the use of tissue microarray or whole tissue sections, the fixation of slides, the use of different primary antibodies, and scoring of the data. Recently, the Lunenburg Lymphoma Biomarker Consortium has been established to unify immunohistochemical methods in biomarker research. So far, the group has focused on standardization and validation of markers with prognostic impact in diffuse large B-cell lymphoma, concentrating especially on variations in techniques and scoring interpretation. ${ }^{25}$ The reproducibility of the results has been observed to be poor, especially for Bcl-6. Furthermore, in a separate study the determination of the germinal center phenotype from the widely used tissue microarray has been observed to be unreliable, due to difficulties in interpretation of Bcl-6 status on tissue microarray in comparison to whole tissue sections. ${ }^{26}$ According to our experience, the staining and interpretation of Bcl- 6 has also been the most challenging.

The aim of this study was to establish an immunohistochemical approach, which could be used as a part of routine diagnostic procedures to classify germinal center and non-germinal center subtypes correctly. To avoid misclassification due to Bcl-6 we focused on the markers related to the activated B-cell-like phenotype. To avoid heterogeneity of the patient cohort we excluded other chemotherapy regimens than R-CHOP. Finally, to take into account the tissue heterogeneity tissue microarrays were excluded and only whole tissue sections of lymphoma samples were accepted in the study.

\section{Materials and methods}

\section{Patients and Treatments}

The retrospectively collected data consisted of $88 \mathrm{de}$ novo CD20-positive diffuse large B-cell lymphoma patients treated between 2002 and 2006 in University Hospitals of Helsinki, Finland, and Lund, Sweden. Cases with primary mediastinal large B-cell lymphoma, primary central nervous system diffuse large B-cell lymphoma, or involvement of low-grade lymphoma were excluded from the cohort. All patients were immunocompetent. The patients were treated uniformly with a combination of rituximab $(\mathrm{R})$ and CHOP regimen (cyclophosphamide, doxorubicin, vincristine, and prednisone). The baseline clinical characteristics were collected and the risk stratification scoring performed applying the International Prognostic Index and revised-International Prognostic Index. ${ }^{27,28}$ The institutional review boards in Helsinki and Lund, and the Finnish National Authority for Medicolegal Affairs approved the study protocol.

\section{Immunohistochemical Stainings}

The immunohistochemical stainings were performed on formalin-fixed, paraffin-embedded $4 \mu \mathrm{m}$ lymphoma tissue sections in Helsinki as described earlier using antibodies for CD10 (1:200; Novocastra Laboratories), Bcl-6 (1:20; Dako Cytomation), MUM1/IRF4 (1:100, Dako Cytomation), Bcl-2 (1:100, Dako Cytomation), and FOXP-1 (1:80; JC12, A Banham). ${ }^{21}$ The slides were scored positive for CD10, Bcl-6, and MUM1/IRF4, if $30 \%$ or more of the lymphoma cells were positive. For Bcl-2 and FOXP1 positivities the cutoff levels were 50 and $100 \%$, 
1096
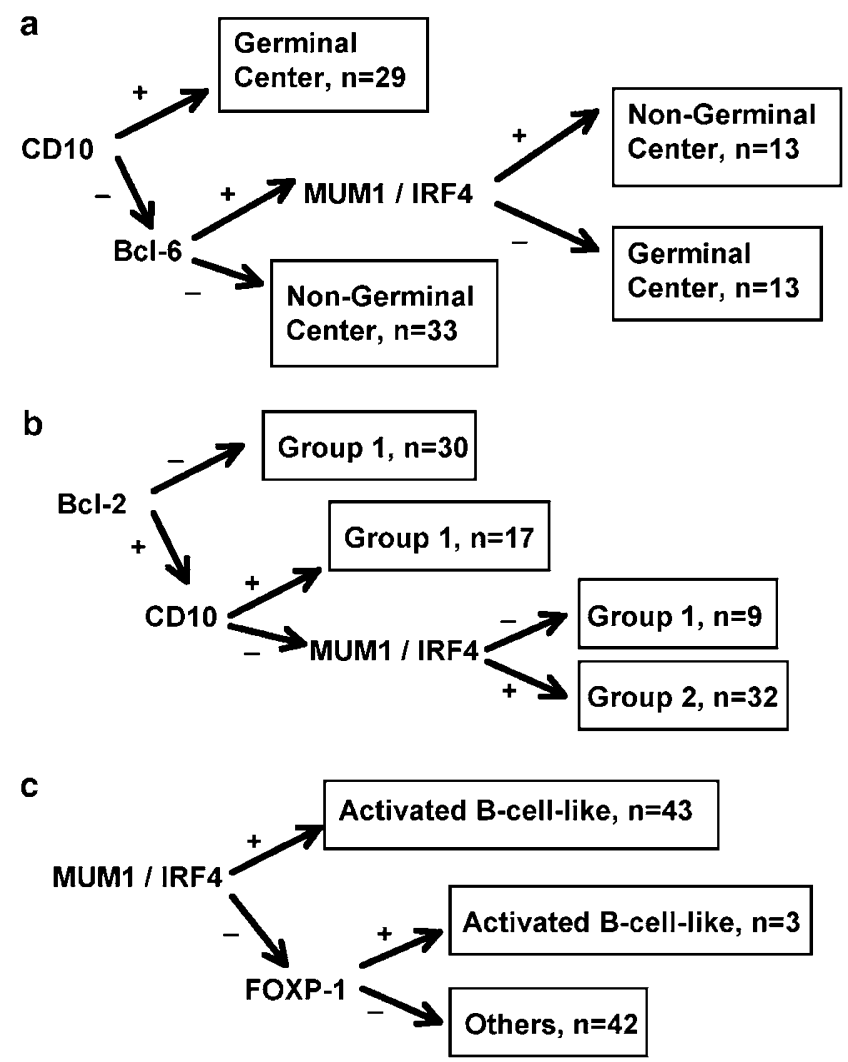

Figure 1 The immunohistochemical classification of the cell of origin. (a) Hans algorithm. ${ }^{11}$ (b) Muris algorithm. ${ }^{24}$ (c) Modified activated B-cell-like algorithm.

respectively. No follicular areas in the histology were analyzed.

Three different algorithms were used (Figure 1). Using the Hans algorithm (Figure 1a), the cases were assigned to germinal center phenotype, if CD10 alone or both CD10 and Bcl-6 were positive. If both CD10 and Bcl-6 were negative, the case was considered to belong to the non-germinal center subgroup. MUM1/IRF4 expression determined the subgroup in cases where CD10 was negative, and Bcl-6 positive: a MUM1/IRF4-positive case was assigned to the non-germinal center subgroup, whereas a MUM1/IRF4-negative case belonged to the germinal center subgroup. According to the Muris algorithm (Figure 1b), the cases were considered to belong to group 1 if Bcl-2 was negative or if Bcl-2 and CD10 were positive. In the cases were Bcl-2 was positive but CD10 negative, the MUM1/ IRF4 expression determined the subgroup: MUM1/ IRF4-negative cases belonged to group 1, whereas MUM1/IRF4-positive cases belonged to group 2. In the modified activated B-cell-like classification (Figure 1c), the cases expressing MUM1/IRF4 or FOXP1 were considered to belong to the activated B-cell-like subgroup, and the rest of the cases were assigned as others.

\section{Statistical Analysis}

Failure-free survival was calculated as the period between diagnosis and date of relapse or death of any cause. Overall survival was determined from the time of diagnosis to death or date of last follow up. The level of agreement between the classifiers was evaluated by Kappa statistics. The Kaplan-Meier method was used to estimate the survival rates and the differences were compared with log-rank test. Chi square test was performed to evaluate the differences in the frequency for the prognostic factors. All $P$-values were two-tailed. A significant level of probability was considered as less than 0.05. The statistical analyses were done with SPSS 14.0 for Windows (SPSS Inc., Chicago, IL, USA).

\section{Results}

\section{Patient Characteristics}

Our cohort included 88 diffuse large B-cell lymphoma patients uniformly treated with the R-CHOP regimen. The patient characteristics are presented in Table 1. Although majority of the patients were older than 60 years and had advanced disease they belonged to the low International Prognostic Index risk group. The patients were further divided into subgroups according to modified activated B-cell-like classification. They were included in the activated B-cell-like group, if either MUM1/IRF4 or FOXP1 expression was positive $(n=46)$. The subgroups were well balanced according to sex, Ann Arbor stage, and International Prognostic Index risk groups. However, patients with the activated B-cell-like phenotype had more often an extranodal involvement in comparison to the other patients $(P=0.059)$. The same difference was also observed with the other algorithms (Hans $P=0.021$, Muris $P=0.002$ ). In addition, with the median age of 66.6 years (range 18-84 years), the patients with the activated B-cell-like phenotype tended to be older than the others. No significant difference was seen if the patients were compared according to age groups $(<60$ vs $\geq 60$ years).

The division of patients according to modified activated B-cell-like classification correlated significantly with all previous characteristics of the immunohistochemically defined germinal center or non-germinal center subtypes; the Hans algorithm $(P<0.001)$, the Muris algorithm $(P<0.001)$, the germinal center-associated markers CD10 $(P<0.001)$, and Bcl-6 $(P=0.001)$. Thirty-three percent positivity of CD10 was similar to previous reports. ${ }^{11,12,14,24,29,30}$ The misclassification rate for the modified classification was $14 \%$ in relation to the Hans algorithm and $16 \%$ in relation to the Muris algorithm. According to Kappa statistics, the mea- 
Table 1 Baseline characteristics of patients according to the modified activated B-cell-like classification

\begin{tabular}{|c|c|c|c|c|}
\hline Characteristics (N) & All & Activated B-cell-like & Others & $\mathrm{P}$-value (all) \\
\hline Patient number & $100 \%(88)$ & $52 \%(46)$ & $48 \%(42)$ & \\
\hline Median age (range, years) & $66.6(18-84)$ & $69.4(23-84)$ & $61.8(18-80)$ & 0.009 \\
\hline \multicolumn{5}{|l|}{ Age } \\
\hline$<60$ years & $28 \%(25)$ & $20 \%(9)$ & $38 \%(16)$ & 0.063 \\
\hline$\geq 60$ years & $72 \%(63)$ & $80 \%(37)$ & $62 \%(26)$ & \\
\hline \multicolumn{5}{|l|}{ Sex } \\
\hline Male & $48 \%(42)$ & $54 \%(25)$ & $40 \%(17)$ & 0.208 \\
\hline Female & $52 \%(46)$ & $46 \%(21)$ & $60 \%(25)$ & \\
\hline \multicolumn{5}{|l|}{ Stage } \\
\hline I-II & $47 \%(41)$ & $46 \%(21)$ & $48 \%(20)$ & 1.000 \\
\hline III-IV & $53 \%(47)$ & $54 \%(25)$ & $52 \%(22)$ & \\
\hline \multicolumn{5}{|l|}{ Extranodal sites } \\
\hline Yes & $49 \%(43)$ & $59 \%(27)$ & $38 \%(16)$ & 0.059 \\
\hline No & $51 \%(45)$ & $41 \%(19)$ & $62 \%(26)$ & \\
\hline \multicolumn{5}{|c|}{ International Prognostic Index } \\
\hline Low, 0-2 & $62 \%(54)$ & $54 \%(25)$ & $71 \%(29)$ & 0.128 \\
\hline High, 3-5 & $38 \%$ (33) & $46 \%$ & $29 \%(12)$ & \\
\hline Missing & 1 & & 1 & \\
\hline \multicolumn{5}{|c|}{ Revised International Prognostic Index } \\
\hline Very good, 0 & $7 \%(6)$ & $2 \%(1)$ & $12 \%(5)$ & 0.088 \\
\hline Good, 1-2 & $55 \%(48)$ & $52 \%(24)$ & $59 \%(24)$ & \\
\hline Poor, $3-5$ & $38 \%(33)$ & $46 \%(21)$ & $29 \%(12)$ & \\
\hline Missing & 1 & & 1 & \\
\hline \multicolumn{5}{|l|}{ Hans algorithm } \\
\hline Germinal center & $48 \%(42)$ & $13 \%(6)$ & $86 \%(36)$ & $<0.001$ \\
\hline Non-germinal center & $52 \%(46)$ & $87 \%(40)$ & $14 \%(6)$ & \\
\hline \multicolumn{5}{|l|}{ Muris algorithm } \\
\hline Group 1 & $64 \%(56)$ & $30 \%(14)$ & $100 \%(42)$ & $<0.001$ \\
\hline Group 2 & $36 \%$ & $70 \%$ & $0 \%(0)$ & \\
\hline
\end{tabular}

sure of agreement value between the modified and Hans classifications was 0.727 .

\section{Survival Analysis}

After a median follow-up time of 37 months, the estimated 3-year failure-free survival was $72 \%$ and 3year overall survival $77 \%$ for all patients. According to International Prognostic Index risk stratification, a significant difference in the outcome was observed between the low-risk (International Prognostic Index score 0-2) and high-risk (International Prognostic Index score 3-5) groups (failure-free survival 84 vs $53 \%, P=0.002$ and overall survival 85 vs $63 \%$, $P=0.012$, Figure 2a and b). As previously shown, revised-International Prognostic Index could also distinguish the patients into three risk groups with very good, good, and poor outcomes (failure-free survival 100 vs 82 vs 53\%, overall survival 100 vs 83 vs $63 \%$, Figure 2c and d). In contrast, no significant difference in the survival was observed between the patients with nodal restricted disease and the ones also having extranodal affisions.
The clinical outcomes according to the modified activated B-cell-like classification are shown in Figure 3a and b. Interestingly, the activated B-celllike group of patients showed a significantly worse failure-free survival and a trend for an inferior overall survival in comparison to the other patients (failurefree survival 63 vs $82 \%, P=0.048$, overall survival 69 vs $85 \%, P=0.110)$. Similarly, the group 2 defined by the Muris algorithm had an adverse outcome compared with the group 1 of patients (failure-free survival 59 vs $81 \%, P=0.041$, overall survival 67 vs $82 \%, P=0.159$; Figure 3c and d). In contrast, when the germinal center and non-germinal center phenotypes were determined according to the Hans algorithm, no difference in survival between the two subgroups was observed (FFS 70 vs $75 \%, P=0.631$, OS 76 vs $77 \%, P=0.894$; Figure $3 e$ and f).

\section{Discussion}

The gene expression signature of diffuse large B-cell lymphoma consists of two major prognostically different subtypes, the germinal center and activated 

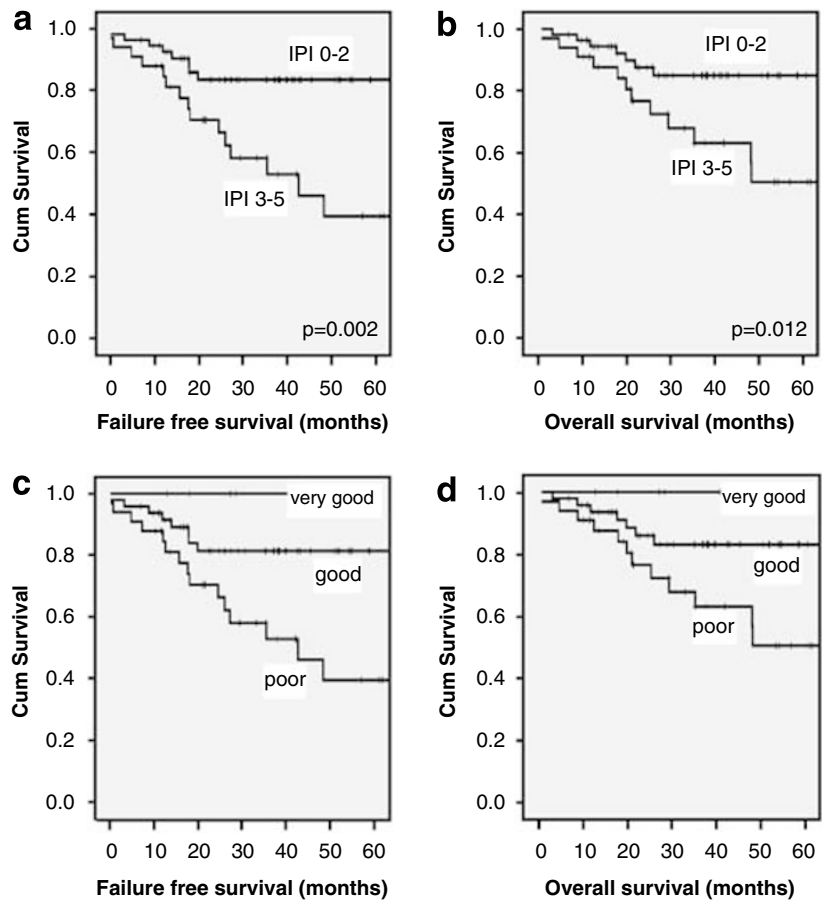

Figure 2 Outcome according to the International Prognostic Index (IPI) and revised International Prognostic Index (R-IPI) risk stratifications. (a) Failure-free survival comparing IPI 0-2 and IPI 3-5 risk groups. (b) Overall survival according to International Prognostic Index. (c) Failure-free survival comparing very good (R-IPI 0), good (R-IPI 1-2), and poor (R-IPI 3-5) risk groups. (d) Overall survival according to revised International Prognostic Index.

B-cell-like phenotypes ${ }^{6-8,10}$ and a third miscellaneous category, type 3 , that also has a poor outcome. ${ }^{7}$ Attempts have been made to reproduce this classification immunohistochemically to facilitate the simple identification of high-risk patients in routine clinical practice. These generally rely on the identification of germinal center vs non-germinal center derived lymphomas rather than the three categories identified by gene expression profiling. Algorithms based on combinations of CD10, Bcl-6, Bcl-2, and MUM1/IRF4 expression have been reported to distinguish two clinically relevant subgroups of patients. ${ }^{11,24,31}$ However, several studies have not been able to confirm the prognostic impact of the algorithms on survival. ${ }^{14-22}$

In this study, we focused on the identification of the markers related to the activated B-cell-like phenotype. The importance of the transcription factors MUM1/IRF4 and FOXP-1 as markers for the activated B-cell-like signature is well established. ${ }^{6,7}$ Likewise, the expression of the anti-apoptotic Bcl-2 has been related to the non-germinal center phenotype. ${ }^{6}$ By using the modified immunohistochemical-activated B-cell-like classification, we could show that the activated B-cell-like subgroup with MUM1/IRF4 and FOXP1 positivity has a significantly inferior failure-free survival in compar- ison to the other diffuse large B-cell lymphoma patients. Furthermore, using the Muris algorithm we found that the patients with Bcl-2-positive, CD10negative, and MUM1/IRF4-positive phenotype (group 2) have an adverse outcome in comparison to the rest of the patients (group 1). However, when the classification of diffuse large B-cell lymphoma was performed according to the Hans algorithm, no difference in the outcome was observed between the subtypes. A minor misclassification rate was demonstrated between the separate algorithms. In our patient cohort, the modified activated B-cell-like classification was more applicable than the Muris algorithm, where the group 2 consisted of more high-risk patients than the group 1 (data not shown). As the classification is based on two markers, it is also technically simpler than the others, as well as more cost effective.

The reason for the difference in the prognostic impact between the activated B-cell-like associated classifications and the Hans algorithm remains to be established, but it may be associated with the Bcl-6 staining, which is a prominent germinal center-associated determinant. In the Hans algorithm, the Bcl-6 staining influences the definition of the phenotype in about $70 \%$ of the CD10-negative cases. There are data indicating that the Bcl-6 stainings are unreliable when they are compared between different units. ${ }^{25}$ Although the distribution of the prognostic markers is very similar in most studies, possible inconsistency follows as an algorithm takes into account all different immunostainings for the individual patient. Therefore, the algorithm should be based on reliable stainings, and the Bcl-6 staining should possibly be excluded. Alternatively, different, more accurate Bcl-6 antibody should be used.

The primary studies describing the Hans and Muris algorithms ${ }^{11,24}$ are based on immunohistochemical analysis made on tissue microarrays. The heterogeneity within the tumor tissue of diffuse large B-cell lymphoma may, however, be underestimated in a tissue microarray that represents only a small proportion of each lymphoma sample. Accordingly, tissue microarray has been reported to be inappropriate for the analysis of Bcl-6 in diffuse large B-cell lymphoma in comparison to staining of whole tissue lymphoma sections. ${ }^{26}$ The explanation for the differing staining results may be due to poor tissue microarray-sampling or heterogeneity of the staining patterns. By comparing these algorithms on whole sections from the same group of patients our study enables a direct comparison of their effectiveness without any bias being introduced through tissue microarray sampling.

The previously reported algorithms and our modified activated B-cell-like classification are attempts to identify the subgroups of diffuse large B-cell lymphoma immunohistochemically. The proposed modified activated B-cell-like classification needs to be tested further in a prospective study to 


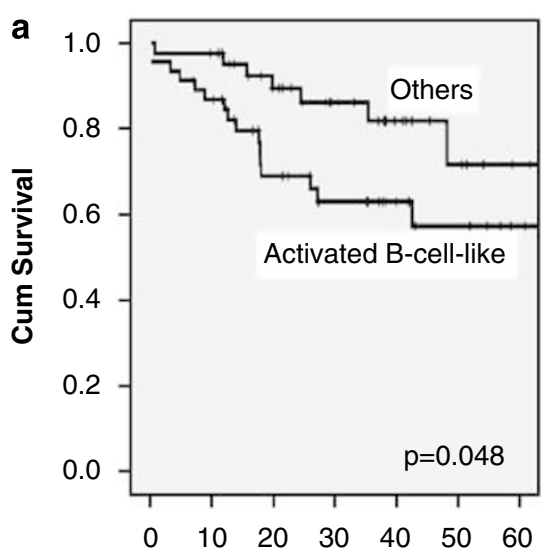

Failure free survival (months)

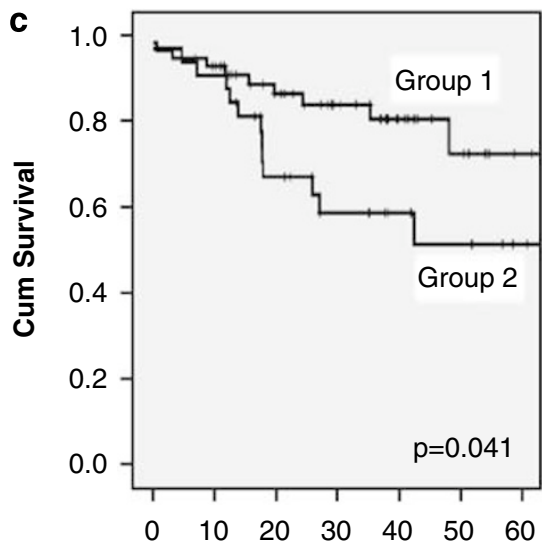

Failure free survival (months)

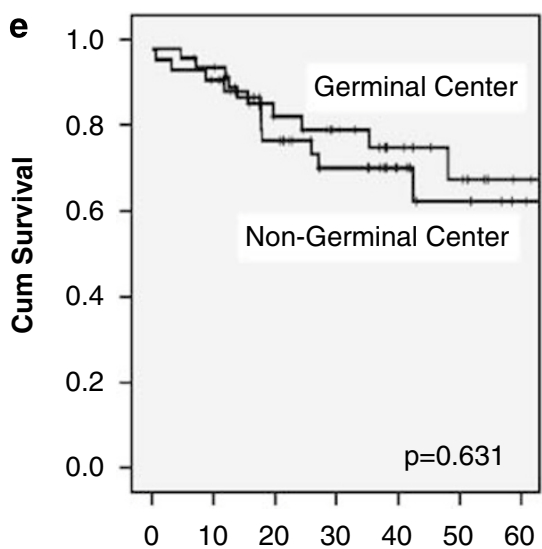

Failure free survival (months)
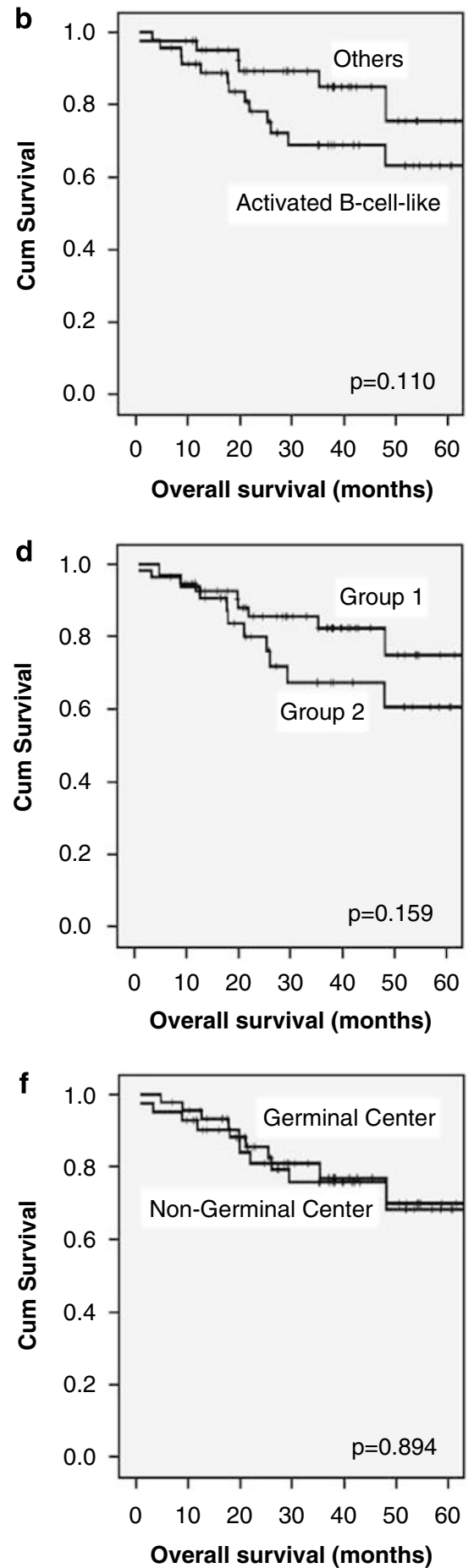

Figure 3 The survival rates according to the modified activated B-cell-like classification, Muris algorithm, and Hans algorithm. (a) Failure-free survival according to the modified activated B-cell-like classification (activated B-cell-like $(n=46)$ vs other $(n=42)$ patients). (b) Overall survival according to the modified activated B-cell-like classification. (c) Failure-free survival according to Muris algorithm (group $1(n=56)$ vs group $2(n=32)$ patients). (d) Overall survival according to the Muris algorithm. (e) Failure-free survival according to the Hans algorithm (germinal center $(n=42)$ vs non-germinal center $(n=46)$ patients). (f) Overall survival according to the Hans algorithm.

confirm the utility of the algorithm. Nevertheless, our data demonstrate that the algorithms based on activated B-cell-like associated markers identify the activated B-cell-like subgroup as an adverse risk factor in immunochemotherapy-treated diffuse large B-cell lymphoma patients. 


\section{Acknowledgements}

The authors thank Onerva Levälampi for technical assistance. The study was supported by grants from the Finnish Academy of Sciences (SL), Finnish Cancer Societies (SL), Sigrid Juselius Foundation (SL), University of Helsinki (SL), Helsinki University Central Hospital (SL and HN), Finnish-Norwegian Medical Foundation (HN), and Bayer Schering Pharma Research Foundation (HN). AHB was supported by the Leukaemia Research Fund.

\section{Conflict of Interest}

The authors declare no conflict of interest.

\section{References}

1 Swerdlow SH, Campo E, Harris NL, et al. WHO Classification of Tumours of Hematopoietic and Lymphoid Tissues. IARCH Press: Lyon, 2008.

2 Coiffier B, Lepage E, Briere J, et al. CHOP chemotherapy plus rituximab compared with CHOP alone in elderly patients with diffuse large-B-cell lymphoma. N Engl J Med 2002;346:235-242.

3 Pfreundschuh M, Ho AD, Cavallin-Stahl E, et al. Prognostic significance of maximum tumour (bulk) diameter in young patients with good-prognosis diffuse large-B-cell lymphoma treated with CHOP-like chemotherapy with or without rituximab: an exploratory analysis of the MabThera International Trial Group (MInT) study. Lancet Oncol 2008;9:435-444.

4 Pfreundschuh $\mathrm{M}$, Schubert J, Ziepert M, et al. Six versus eight cycles of bi-weekly CHOP-14 with or without rituximab in elderly patients with aggressive CD20+ B-cell lymphomas: a randomised controlled trial (RICOVER-60). Lancet Oncol 2008;9:105-116.

5 Sehn LH, Donaldson J, Chhanabhai M, et al. Introduction of combined CHOP plus rituximab therapy dramatically improved outcome of diffuse large B-cell lymphoma in British Columbia. J Clin Oncol 2005;23:5027-5033.

6 Alizadeh AA, Eisen MB, Davis RE, et al. Distinct types of diffuse large B-cell lymphoma identified by gene expression profiling. Nature 2000;403:503-511.

7 Rosenwald A, Wright G, Chan WC, et al. The use of molecular profiling to predict survival after chemotherapy for diffuse large-B-cell lymphoma. N Engl J Med 2002;346:1937-1947.

8 Jais JP, Haioun C, Molina TJ, et al. The expression of 16 genes related to the cell of origin and immune response predicts survival in elderly patients with diffuse large B-cell lymphoma treated with CHOP and rituximab. Leukemia 2008;22:1917-1924.

9 Rimsza LM, Leblanc ML, Unger JM, et al. Gene expression predicts overall survival in paraffinembedded tissues of diffuse large B-cell lymphoma treated with R-CHOP. Blood 2008;112:3425-3433.

10 Lenz G, Wright G, Dave SS, et al. Stromal gene signatures in large-B-cell lymphomas. $\mathrm{N}$ Engl J Med 2008;359:2313-2323.

11 Hans CP, Weisenburger DD, Greiner TC, et al. Confirmation of the molecular classification of diffuse large B-cell lymphoma by immunohistochemistry using a tissue microarray. Blood 2004;103:275-282.

12 Berglund M, Thunberg U, Amini RM, et al. Evaluation of immunophenotype in diffuse large B-cell lymphoma and its impact on prognosis. Mod Pathol 2005;18: 1113-1120.

13 Sjo LD, Poulsen CB, Hansen M, et al. Profiling of diffuse large B-cell lymphoma by immunohistochemistry: identification of prognostic subgroups. Eur J Haematol 2007;79:501-507.

14 Colomo L, Lopez-Guillermo A, Perales $\mathrm{M}$, et al. Clinical impact of the differentiation profile assessed by immunophenotyping in patients with diffuse large B-cell lymphoma. Blood 2003;101:78-84.

15 De Paepe P, Achten R, Verhoef G, et al. Large cleaved and immunoblastic lymphoma may represent two distinct clinicopathologic entities within the group of diffuse large B-cell lymphomas. J Clin Oncol 2005;23:7060-7068.

16 Moskowitz CH, Zelenetz AD, Kewalramani $\mathrm{T}$, et al. Cell of origin, germinal center versus nongerminal center, determined by immunohistochemistry on tissue microarray, does not correlate with outcome in patients with relapsed and refractory DLBCL. Blood 2005;106:3383-3385.

17 Nyman H, Adde M, Karjalainen-Lindsberg ML, et al. Prognostic impact of immunohistochemically defined germinal center phenotype in diffuse large B-cell lymphoma patients treated with immunochemotherapy. Blood 2007;109:4930-4935.

18 Wilson WH, Dunleavy K, Pittaluga S, et al. Phase II study of dose-adjusted EPOCH and rituximab in untreated diffuse large B-cell lymphoma with analysis of germinal center and post-germinal center biomarkers. J Clin Oncol 2008;26:2717-2724.

19 Saito B, Shiozawa E, Usui T, et al. Rituximab with chemotherapy improves survival of non-germinal center type untreated diffuse large B-cell lymphoma. Leukemia 2007;21:2563-2566.

20 Uccella S, Placidi C, Marchet S, et al. Bcl-6 protein expression, and not the germinal centre immunophenotype, predicts favourable prognosis in a series of primary nodal diffuse large B-cell lymphomas: a single centre experience. Leuk Lymphoma 2008;49:1321-1328.

21 Nyman H, Jerkeman M, Karjalainen-Lindsberg ML, et al. Bcl-2 but not FOXP1, is an adverse risk factor in immunochemotherapy treated non-germinal center diffuse large B-cell lymphomas. Eur J Haematol 2009;82:364-372.

22 Song MK, Chung JS, Shin DH, et al. Prognostic significance of the Bcl-2 negative germinal centre in patients with diffuse large B cell lymphoma treated with R-CHOP. Leuk Lymphoma 2009;50:54-61.

$23 \mathrm{Fu} \mathrm{K}$, Weisenburger DD, Choi WW, et al. Addition of rituximab to standard chemotherapy improves the survival of both the germinal center B-cell-like and non-germinal center B-cell-like subtypes of diffuse large B-cell lymphoma. J Clin Oncol 2008;26: 4587-4594.

24 Muris JJ, Meijer CJ, Vos W, et al. Immunohistochemical profiling based on Bcl-2, CD10 and MUM1 expression improves risk stratification in patients with primary nodal diffuse large $B$ cell lymphoma. J Pathol 2006;208:714-723.

25 de Jong D, Xie W, Rosenwald A, et al. Immunohistochemical prognostic markers in diffuse large B-cell lymphoma: validation of tissue microarray as a 
prerequisite for broad clinical applications. A study from the Lunenburg Lymphoma Biomarker Consortium (LLBC). J Clin Pathol 2009;62:128-138.

26 Linderoth J, Ehinger M, Akerman M, et al. Tissue microarray is inappropriate for analysis of BCL6 expression in diffuse large B-cell lymphoma. Eur J Haematol 2007;79:146-149.

27 A predictive model for aggressive non-Hodgkin's lymphoma. The International Non-Hodgkin's Lymphoma Prognostic Factors Project. N Engl J Med 1993;329: 987-994.

28 Sehn LH, Berry B, Chhanabhai M, et al. The revised International Prognostic Index (R-IPI) is a better predictor of outcome than the standard IPI for patients with diffuse large B-cell lymphoma treated with RCHOP. Blood 2007;109:1857-1861.

29 Lossos IS, Jones CD, Warnke R, et al. Expression of a single gene, BCL-6, strongly predicts survival in patients with diffuse large B-cell lymphoma. Blood 2001;98:945-951.

30 Fabiani B, Delmer A, Lepage E, et al. CD10 expression in diffuse large B-cell lymphomas does not influence survival. Virchows Arch 2004;445:545-551.

31 Amen F, Horncastle D, Elderfield K, et al. Absence of cyclin-D2 and Bcl-2 expression within the germinal centre type of diffuse large B-cell lymphoma identifies a very good prognostic subgroup of patients. Histopathology 2007;51:70-79. 